\title{
Effective anti-tuberculosis therapy correlates with plasma small RNA
}

\begin{abstract}
To the Editor:
Small RNA (smRNA) is a diverse family of translation regulation molecules, which includes microRNA (miRNA). Changes in miRNA have been reported in association with respiratory disease [1] and in mycobacterial infection [2]. Studies have measured concentrations of miRNA pooled from multiple subjects with active tuberculosis (TB) disease compared with latently infected individuals and healthy controls [3]. However, this approach cannot assess variation between individuals, nor does it describe changes that might occur with treatment, both of which may be helpful in characterising and predicting patient outcome.
\end{abstract}

Here, we investigated plasma host smRNA in adults being treated for pulmonary TB. 41 subjects with confirmed smear-positive pulmonary TB were recruited prospectively in Durban, South Africa. Three were excluded due to missing time-points during follow-up. 29 (76.3\%) out of 38 subjects were male and 19 (50\%) out of 38 were HIV-1 co-infected. Treatment was with a standard drug regimen for pulmonary TB: 2 months of isoniazid (INH), rifampicin (RIF), ethambutol and pyrazinamide, followed by 4 months of INH and RIF. At day 0 (pretreatment) and week 24 (treatment completion) each patient had $50 \mathrm{~mL}$ whole blood collected into K3-EDTA vacutainers (BD, Gauteng, South Africa) by venepuncture. Plasma was frozen at $-80^{\circ} \mathrm{C}$ until required. This study was approved by the Biomedical Research Ethics Committee of the University of Kwa-Zulu Natal (approval number: BF 138/09); and conducted in compliance with ICH Good Clinical Practice Guidelines. All patients provided informed consent.

Haemolysis has been found to affect plasma miRNA concentration if absorbance at $414 \mathrm{~nm}\left(\mathrm{~A}_{414}\right)$ is $>0.2$ at $[4,5]$. We measured haemoglobin $(\mathrm{Hb})$ according to Harboe [6] and excluded paired samples with $\mathrm{Hb}$ differences $>0.5 \mathrm{~g} \cdot \mathrm{L}^{-1}$ (equivalent to an absorbance of 0.2 at $\mathrm{A}_{414}$ in our system), which made up four $(10.5 \%)$ out of the 38 subjects.

smRNA was extracted from $200 \mu \mathrm{L}$ plasma before and after anti-TB treatment ( $\mathrm{n}=34$; 17 with HIV-1 co-infection) using the miRNeasy mini kit (Qiagen, Germantown, MD, USA), according to the manufacturer's instructions. 30 individuals were culture-negative at week 24, 14 of whom were HIV-1 co-infected (all patients produced sputum at week 24 and this was tested). Four individuals had sputum that was still culture-positive at week 24, three of whom were HIV-1 co-infected. Of these, one had drug-sensitive TB, one had INH resistance and two had multidrug-resistant (MDR)-TB.

Plasma smRNA concentration (6-150 nucleotides) was determined using the 2100 Bioanalyzer (Agilent Technologies, Santa Clara, CA, USA). Those who culture-converted had significantly higher plasma smRNA (6-150 nucleotides) pretreatment (day 0) compared with post-treatment (week 24) (day 0 median (interquartile range (IQR)) $353(238-573) \mathrm{pg} \cdot \mu \mathrm{L}^{-1}$, week 24 median (IQR) $83(53-146) \mathrm{pg} \cdot \mu \mathrm{L}^{-1}$; $\mathrm{p}<0.0001$ ) (fig. 1a). Individuals who failed therapy $(n=4)$ did not have a similar decline in smRNA (day 0 median (IQR) 115 (86-515) pg. $\mu \mathrm{L}^{-1}$, week 24 median (IQR) 348 (165-554) pg. $\mu \mathrm{L}^{-1}$; p>0.05) (fig. 1b). Absolute measures of smRNA in plasma were variable between patients, precluding use of absolute concentrations to inform disease status. Despite small numbers there was a significant difference in plasma smRNA concentration before and after treatment for those who culture-converted compared with treatment failures $(\geqslant 10 \%$ increase or decline in smRNA concentration between the two time-points was considered a change; $\mathrm{p}=0.02$ using Fisher's exact test).

Host nucleic acid in serum was first noted in 1978 [7], but its function and source remain unknown. We found no correlation between blood $\mathrm{CD}^{+}{ }^{+} \mathrm{T}$-cell count and concentration of smRNA at day 0 for HIV-positive subjects (Spearman rank correlation $\mathrm{r}=0.25$, $\mathrm{p}=$ nonsignificant; data not shown), suggesting $\mathrm{CD} 4^{+} \mathrm{T}$-cells are not the primary source of plasma smRNA. This does not rule out production from other blood cells or disease site.

Co-infection with HIV-1 did not affect decline in plasma smRNA after anti-TB treatment (both $\mathrm{p}<0.001$ ) (fig. 1c, d); and there was no significant difference in smRNA between HIV-1 infected or uninfected subjects at day 0 or week 24 (both $\mathrm{p}>0.05$ ) (fig. 1c, d). 
a)

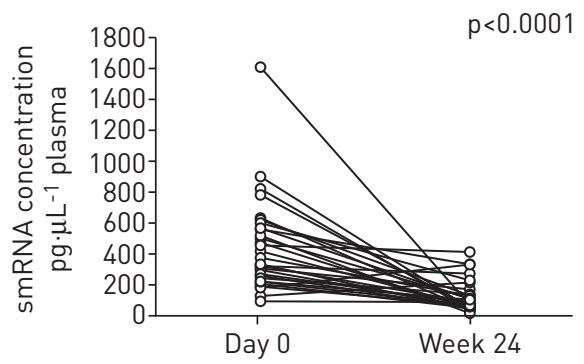

c)

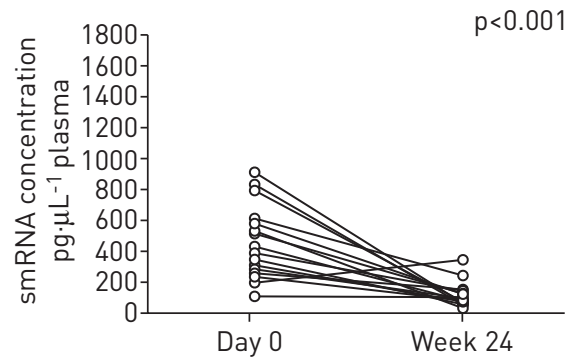

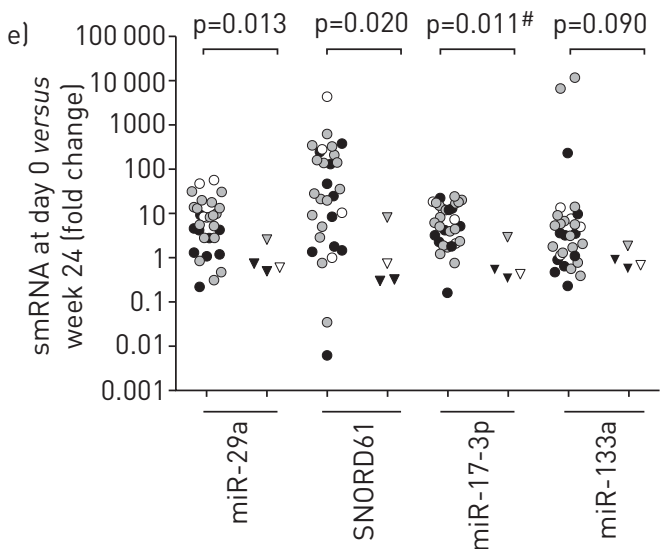

b)



d)

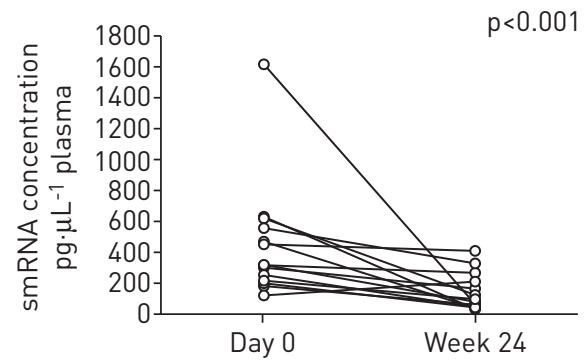

f)

\begin{tabular}{lcc}
\hline \multicolumn{3}{c}{$\begin{array}{c}\text { Fold change day 0 compared with week 24 } \\
\text { Median (interquartile range) }\end{array}$} \\
\hline & $\begin{array}{c}\text { Culture negative } \\
\text { at week 24 }\end{array}$ & $\begin{array}{c}\text { Culture positive } \\
\text { at week 24 }\end{array}$ \\
miR-29A & $6.01(2.12-11.78)$ & $-1.74(-2.23-1.30)$ \\
SNORD61 & $23.24(2.28-194)$ & $-2.60(-3.80-4.94)$ \\
miR-17-3p & $4.98(1.95-13.66)$ & $-2.42(-3.18-1.38)$ \\
miR-133a & $2.94(-1.10-7.01)$ & $-1.48(-1.92-0.90)$ \\
\hline
\end{tabular}

g)

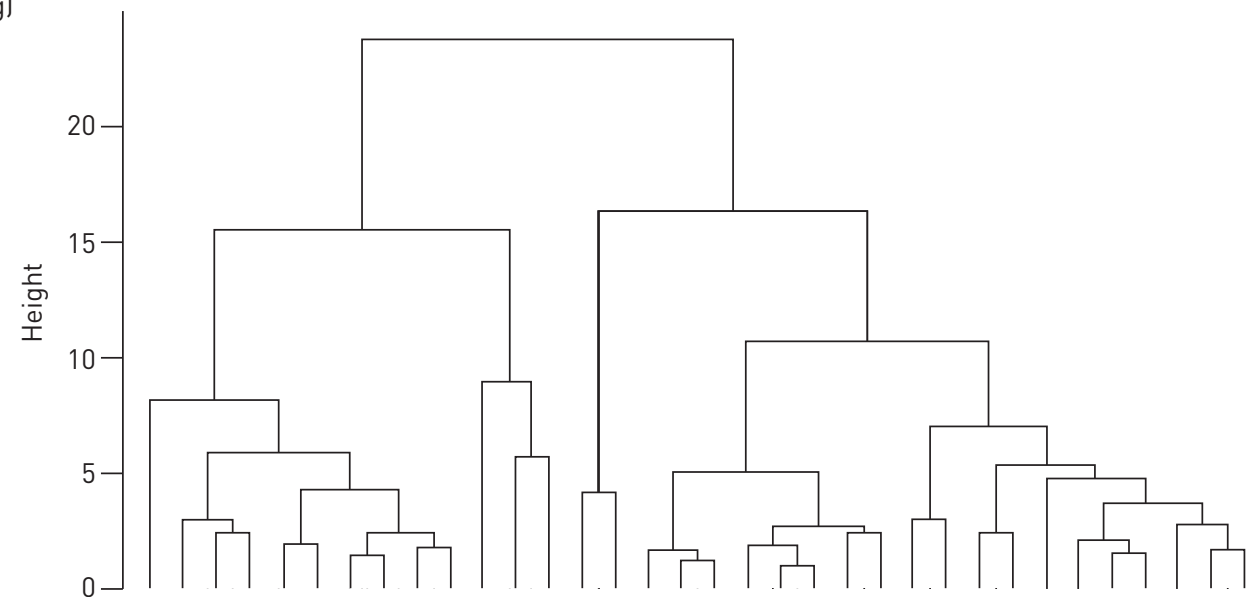

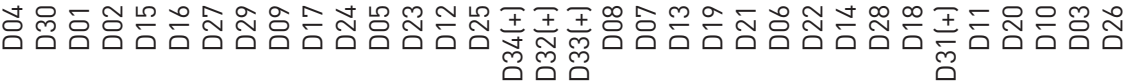

FIGURE 1 Small RNA (smRNA) profiles in plasma of tuberculosis (TB) infected individuals before and after treatment. a-d) Wilcoxon paired analysis of plasma smRNA concentration (6-150 nucleotides) before (day 0) and after (week 24) anti-TB treatment for individuals with active TB. a) Individuals who were culture negative at week $24(\mathrm{n}=30)$ and $\mathrm{b})$ who were culture positive at week $24(\mathrm{n}=4)$. smRNA according to HIV status: c) HIV-negative individuals; and d) HIV co-infected individuals. Ns: nonsignificant. e, f) Fold change for four individual smRNA genes at day 0 (pretreatment) compared with week 24 (after treatment) in those that culture converted by week 24 (circles, $n=30$ ) compared with those who were culture-positive at week 24 (triangles, $\mathrm{n}=4$ ). p-values are uncorrected. \#: indicates statistical significance after Bonferroni correction. Grey symbols: HIV-negative; black symbols: co-infected with HIV-1, antiretroviral therapy naïve; white symbols: co-infected with HIV-1, receiving antiretroviral therapy. g) Cluster diagram using the four individually tested microRNA species (miR-29a, 17-3p, 133a, and SNORD61). (+): indicates those who were culture-positive after treatment completion. 
Rotorgene-100 human serum/plasma MIHS-106Z arrays (SABiosciences, Germantown, MD, USA) were used to measure 84 miRNAs and five small nucleolar RNAs (snoRNAs). Day 0 and week 24 miRNA was converted to cDNA for eight individuals (five HIV-negative individuals and three HIV-1 co-infected) and tested on individual arrays. Mean fold changes for each smRNA were determined using the previously described $2^{-\Delta \Delta C T}$ method $[8,9]$. No detection for a smRNA was assigned a cycle threshold of 40 . Cycles $<40$ were included and considered positive.

20 out of 90 tested smRNAs were raised at day 0 compared with week 24 and none decreased ( $\geqslant 2$-fold, $\mathrm{p}<0.05$; data not shown). After correction all statistical significance was lost indicating measurement of any individual smRNA was no more discriminatory than total smRNA concentration. In a previous study, pooled serum from Mycobacterium tuberculosis-infected individuals, compared with healthy controls, had 59 out of 1223 miRNAs increased and 33 decreased in the active TB group [3]. A study of paediatric TB observed miR-21 and miR-26a increased in peripheral blood in response to treatment [10]. That we found no miRNA molecules increased in response to therapy may reflect our measurement of miRNA in plasma rather than in $\mathrm{CD}^{+}$T-cells [10]. A study comparing peripheral blood and pleural fluid in TB-infected individuals suggested a differential compartmentalisation of miRNA at the disease site compared with peripheral blood [11].

Despite a previous reported association between active TB and increased miR-29a in serum [3], and a potential mechanism due to interaction with the interferon- $\gamma$ gene [12], we found, similar to miR-133a and SNORD61, only a trend towards a difference in fold change for miR-29a in those who culture-converted $(n=30)$ compared with those who did not $(n=4)$ (fig. 1e, f). Change in miR-17-3p was weakly statistically significant after correction ( $p=0.04)$. There was a large inter-patient range in smRNA fold change between individuals for all smRNAs tested. It is possible this could reflect differences such as sterilising cure versus return to latency, high variability in the immune response or the severity of disease at clinic presentation.

HIV status did not appear to affect our observations, whereas a previous report found some miRNAs associated with high HIV-1 viral load in individuals without active TB [13]. Most HIV-1 co-infected subjects in our study were antiretroviral therapy (ART) naïve, suggesting viral suppression is not a prerequisite for decline in smRNA at TB treatment completion. Although only five (29\%) out of $17 \mathrm{HIV}-1$ co-infected individuals were using ART, there appeared to be no significant impact on detection of SNORD61, miR-17-3p or miR-133a compared with either HIV-negative or HIV-positive but ARV naïve subjects. For miR-29a, those who were both on ART and culture-negative at week 24 all had fold changes above the median.

snoRNAs have been characterised as a structural scaffold for ribosome assembly [14]. SnoRNAs have not been previously investigated in $M$. tuberculosis infection and snoRNA would not be expected to be present outside of the nucleolus. However, several snoRNA molecules have been observed to accumulate in the cytosol in times of cellular stress [15], which may suggest additional functions for these molecules.

We performed cluster analysis to test whether using multiple smRNA expression ratios (miR-17-3p, SNORD61, miR-29a and miR-133a) would increase the utility of smRNA for determining culture status at week 24. Three of the subjects (D32, D33 and D34) who were still culture positive at week 24 of treatment formed a distinct cluster (fig. $1 \mathrm{~g}$ ). All had INH or MDR-TB drug-resistance. D31 did not cluster with the other treatment failures and was found to have drug-sensitive TB. The significance of this is uncertain as miRNA profiles identified as being associated with active TB are nonspecific as they overlap with other respiratory diseases [16]. Our study is limited by the small numbers who remained culture-positive following treatment. In addition, determining the rate and pattern of decline in smRNA in subjects with different treatment responses would provide further evidence of the value of smRNA as an early indicator of outcome.

In summary, we find changes in plasma smRNA concentration are predictive of individuals who have responded to anti-TB treatment at week 24, independent of HIV-1 co-infection, and that measurement of total smRNA or a combination of smRNA markers present in plasma may be a useful marker of treatment response in active TB. Such markers could potentially be utilised to provide a rapid indication of therapy success or failure in the clinic.

@ERSpublications

Plasma small RNA concentration declines in response to anti-tuberculosis therapy and is independent of HIV-status http://ow.ly/IxOaD

Isobella Honeyborne', Marc C. Lipman ${ }^{2}$, Clare Eckold ${ }^{1}$, Dimitrios Evangelopoulos ${ }^{1}$, Stephen H. Gillespie ${ }^{3}$, Alexander $\mathrm{Pym}^{4}$ and Timothy D. McHugh ${ }^{1}$

${ }^{1}$ Centre for Clinical Microbiology, Dept of Infection, University College London, London, UK. ${ }^{2}$ Centre for Respiratory Medicine, University College London, London, UK. ${ }^{3}$ School of Medicine, Medical and Biological Sciences, University of St Andrews, St Andrews, UK. ${ }^{4}$ K-RITH (KwaZulu-Natal Research Institute for Tuberculosis and HIV), Nelson R. Mandela School of Medicine, University of KwaZulu-Natal, Durban, South Africa. 
Correspondence: Isobella Honeyborne, Centre for Clinical Microbiology, Dept of Infection, University College London, Royal Free Campus, Rowland Hill Street, London, NW3 2PF, UK. E-mail: i.honeyborne@ucl.ac.uk

Received: Sept 212014 | Accepted after revision: Jan 152015 | First published online: March 052015

Support statement: This work was supported in part by the Medical Research Council (grant number: G0601466). M.C. Lipman is a Higher Education Funding Council for England Clinical Senior Lecturer. Funding information for this article has been deposited with FundRef

Conflict of interest: Disclosures can be found alongside the online version of this article at erj.ersjournals.com

Acknowledgements: We would like to thank S. Bokhari and S. Christou (Centre for Clinical Microbiology, Dept of Infection, University College London, London, UK) for assistance with sample processing and C. Smith (Research Dept of Infection and Population Health, University College London, London, UK) for statistical support.

\section{References}

Rupani H, Sanchez-Elsner T, Howarth P. MicroRNAs and respiratory diseases. Eur Respir J 2013; 41: 695-705.

Singh PK, Singh AV, Chauhan DS. Current understanding on micro RNAs and its regulation in response to mycobacterial infections. J Biomed Sci 2013; 20: 14.

$3 \mathrm{Fu} \mathrm{Y}, \mathrm{Yi} \mathrm{Z}, \mathrm{Wu} \mathrm{X}$, et al. Circulating microRNAs in patients with active pulmonary tuberculosis. J Clin Microbiol 2011; 49: 4246-4251.

4 Kirschner MB, Edelman JJ, Kao SC, et al. The impact of hemolysis on cell-free microRNA biomarkers. Front Genet 2013; 4: 94

5 Kirschner MB, Kao SC, Edelman JJ, et al. Haemolysis during sample preparation alters microRNA content of plasma. PLoS One 2011; 6: e24145.

6 Harboe M. A method for determination of hemoglobin in plasma by near-ultraviolet spectrophotometry. Scand J Clin Lab Invest 1959; 11: 66-70.

7 Stroun M, Anker P, Beljanski M, et al. Presence of RNA in the nucleoprotein complex spontaneously released by human lymphocytes and frog auricles in culture. Cancer Res 1978; 38: 3546-3554.

8 Livak KJ, Schmittgen TD. Analysis of relative gene expression data using real-time quantitative PCR and the $2^{-\Delta \Delta C(T)}$ method. Methods 2001; 25: 402-408.

9 Applied Biosystems. User Bulletin \#2 ABI PRISM 7700 Sequence Detection System. www3.appliedbiosystems.com/ cms/groups/mcb_support/documents/generaldocuments/cms_040980.pdf Date last updated: October 2001.

10 Kleinsteuber K, Heesch K, Schattling S, et al. Decreased expression of miR-21, miR-26a, miR-29a, and miR-142-3p in CD4 ${ }^{+} \mathrm{T}$ cells and peripheral blood from tuberculosis patients. PLoS One 2013; 8: e61609.

11 Spinelli SV, Diaz A, D'Attilio L, et al. Altered microRNA expression levels in mononuclear cells of patients with pulmonary and pleural tuberculosis and their relation with components of the immune response. Mol Immunol 2013; 53: 265-269.

12 Ma F, Xu S, Liu X, et al. The microRNA miR-29 controls innate and adaptive immune responses to intracellular bacterial infection by targeting interferon-gamma. Nat Immunol 2011; 12: 861-869.

13 Duskova K, Nagilla P, Le HS, et al. MicroRNA regulation and its effects on cellular transcriptome in human immunodeficiency virus-1 (HIV-1) infected individuals with distinct viral load and CD4 cell counts. BMC Infect Dis 2013; 13: 250 .

14 Williams GT, Farzaneh F. Are snoRNAs and snoRNA host genes new players in cancer? Nat Rev Cancer 2012; 12 : $84-88$.

15 Michel CI, Holley CL, Scruggs BS, et al. Small nucleolar RNAs U32a, U33, and U35a are critical mediators of metabolic stress. Cell Metab 2011; 14: 33-44.

16 Maertzdorf J, Weiner J 3rd, Mollenkopf HJ, et al. Common patterns and disease-related signatures in tuberculosis and sarcoidosis. Proc Natl Acad Sci USA 2012; 109: 7853-7858. 